University of Wollongong

Research Online

Australian Institute for Innovative Materials -

Papers

Australian Institute for Innovative Materials

$1-1-2013$

Highly water-soluble magnetic iron oxide (Fe304) nanoparticles for drug delivery: enhanced in vitro therapeutic efficacy of doxorubicin and MION conjugates

Muhammad Irfan Majeed

Huazhong University Of Science And Technology

Qunwei Lu

Huazhong University Of Science \& Technology

Wei Yan

Huazhong University Of Science \& Technology

Zhen Li

University of Wollongong, zhenl@uow.edu.au

Irshad Hussain

Lahore University of Management Sciences, Pakistan

See next page for additional authors

Follow this and additional works at: https://ro.uow.edu.au/aiimpapers

Part of the Engineering Commons, and the Physical Sciences and Mathematics Commons

Research Online is the open access institutional repository for the University of Wollongong. For further information contact the UOW Library: research-pubs@uow.edu.au 


\title{
Highly water-soluble magnetic iron oxide (Fe304) nanoparticles for drug delivery: enhanced in vitro therapeutic efficacy of doxorubicin and MION conjugates
}

\author{
Abstract \\ We report a simple one step protocol for the preparation of fairly monodisperse and highly water-soluble \\ magnetic iron oxide nanoparticles (MIONs) through a co-precipitation method using a novel \\ multifunctional, biocompatible and water-soluble polymer ligand dodecanethiol-polymethacrylic acid \\ (DDT-PMAA). DDT-PMAA owing to its several intrinsic properties, not only efficiently controls the size of \\ the MIONs but also gives them excellent water solubility, long time stability against aggregation and \\ oxidation, biocompatibility and multifunctional surface rich in thioether and carboxylic acid groups. The \\ molecular weight and concentration of the polymer ligand were optimized to produce ultrasmall (4.6+/- \\ $0.7 \mathrm{~nm}$ ) MIONs with high magnetization (50 emu g(-1)). The MIONs obtained with $1.5 \mathrm{mM}$ DDT-PMAA \\ $(5330 \mathrm{~g} \mathrm{~mol}(-1))$ are highly stable in solution as well as in dry powder form for an extended period of time. \\ These MIONs show a high degree of monodispersity and are superparamagnetic at room temperature. \\ The polymer ligand and MIONs@Polymer were characterized by GPC, H-1 NMR, DLS, TEM, FTIR-Raman, \\ XRD, TGA and VSM. In order to demonstrate the bio-applications of these magnetic nanoparticles (NPs), \\ their toxicity was determined by MTT assay and they were found to be non-toxic and biocompatible. \\ Finally, MIONs were conjugated with the anti-cancer drug doxorubicin (DOX) and its efficacy, as a model \\ drug delivery system, was determined using HepG2 cells. The efficiency of the drug-NP conjugates i.e., \\ covalently bound DOX-MIONs and electrostatically loaded DOX/MIONs, was found to be significantly \\ higher than that of the free drug (DOX).
}

\section{Keywords}

doxorubicin, oxide, iron, mion, magnetic, conjugates, soluble, water, highly, therapeutic, delivery, enhanced, vitro, efficacy, drug, nanoparticles, fe3o4

\section{Disciplines}

Engineering | Physical Sciences and Mathematics

\section{Publication Details}

Majeed, M. Irfan., Lu, Q., Yan, W., Li, Z., Hussain, I., Tahir, M. Nawaz., Tremel, W. \& Tan, B. (2013). Highly water-soluble magnetic iron oxide (Fe304) nanoparticles for drug delivery: enhanced in vitro therapeutic efficacy of doxorubicin and MION conjugates. Journal Of Materials Chemistry B, 1 (22), 2874-2884.

\section{Authors}

Muhammad Irfan Majeed, Qunwei Lu, Wei Yan, Zhen Li, Irshad Hussain, Muhammad Nawaz Tahir, Wolfgang Tremel, and Bien Tan 
Cite this: J. Mater. Chem. B, 2013, 1, 2874

Received 8th March 2013 Accepted 11th April 2013

DOI: $10.1039 / \mathrm{c} 3 \mathrm{tb} 20322 \mathrm{k}$

www.rsc.org/MaterialsB

\section{Highly water-soluble magnetic iron oxide $\left(\mathrm{Fe}_{3} \mathrm{O}_{4}\right)$ nanoparticles for drug delivery: enhanced in vitro therapeutic efficacy of doxorubicin and MION conjugates $\uparrow$}

\author{
Muhammad Irfan Majeed, ${ }^{a}$ Qunwei Lu, ${ }^{\text {b }}$ Wei Yan, ${ }^{\text {af }}$ Zhen Li, ${ }^{c}$ Irshad Hussain, ${ }^{* d}$ \\ Muhammad Nawaz Tahir, ${ }^{e}$ Wolfgang Tremel ${ }^{e}$ and Bien Tan*a
}

\begin{abstract}
We report a simple one step protocol for the preparation of fairly monodisperse and highly water-soluble magnetic iron oxide nanoparticles (MIONs) through a co-precipitation method using a novel multifunctional, biocompatible and water-soluble polymer ligand dodecanethiol-polymethacrylic acid (DDT-PMAA). DDT-PMAA owing to its several intrinsic properties, not only efficiently controls the size of the MIONs but also gives them excellent water solubility, long time stability against aggregation and oxidation, biocompatibility and multifunctional surface rich in thioether and carboxylic acid groups. The molecular weight and concentration of the polymer ligand were optimized to produce ultrasmall ( $4.6 \pm$ $0.7 \mathrm{~nm}$ ) MIONs with high magnetization $\left(50 \mathrm{emu} \mathrm{g}^{-1}\right.$ ). The MIONs obtained with $1.5 \mathrm{mM}$ DDT-PMAA $\left(5330 \mathrm{~g} \mathrm{~mol}^{-1}\right)$ are highly stable in solution as well as in dry powder form for an extended period of time. These MIONs show a high degree of monodispersity and are superparamagnetic at room temperature. The polymer ligand and MIONs@Polymer were characterized by GPC, ${ }^{1} \mathrm{H}$ NMR, DLS, TEM, FTIR-Raman, XRD, TGA and VSM. In order to demonstrate the bio-applications of these magnetic nanoparticles (NPs), their toxicity was determined by MTT assay and they were found to be non-toxic and biocompatible. Finally, MIONs were conjugated with the anti-cancer drug doxorubicin (DOX) and its efficacy, as a model drug delivery system, was determined using HepG2 cells. The efficiency of the drugNP conjugates i.e., covalently bound DOX-MIONs and electrostatically loaded DOX/MIONs, was found to be significantly higher than that of the free drug (DOX).
\end{abstract}

\section{Introduction}

${ }^{a}$ Hubei Key Laboratory of Material Chemistry and Service Failure, Key Laboratory for Large-Format Battery Materials and System, Ministry of Education,School of Chemistry and Chemical Engineering, Huazhong University of Science and Technology, Wuhan, P.R. China. E-mail: bien.tan@mail.hust.edu.cn; Fax: +86 27 87543632; Tel: +862787558172

${ }^{b}$ Key Laboratory of Molecular Biophysics of Ministry of Education, School of Life Science and Technology, Huazhong University of Science and Technology, Wuhan, P.R. China

'Institute for Superconducting and Electronic Materials, Australian Institute of Innovative Materials, University of Wollongong, Australia

${ }^{d}$ Department of Chemistry, SBA School of Science \& Engineering (SSE), Lahore University of Management Sciences (LUMS), DHA, Lahore Cantt-54792, Pakistan. E-mail: ihussain@lums.edu.pk; Tel: +924235608133

'Institute for Inorganic and Analytical Chemistry, University of Mainz, Duesbergweg 10-14, 55128 Mainz, Germany

${ }^{f}$ Faculty of Materials Science and Engineering, Hubei University, Wuhan, P. R. China. E-mail: willieyancn2003@gmail.com

$\dagger$ Electronic supplementary information (ESI) available: Details of synthesis and characterization of polymer ligands with the reaction scheme, gel permeation chromatography and ${ }^{1} \mathrm{H}$ NMR spectroscopy results, MION synthesis procedure and characterization results such as TEM images, UV-visible, FTIR, XRD, Raman spectra, TGA and VSM, cytotoxicity analysis, $\mathrm{pH}$ and salt stability tests etc. See DOI: $10.1039 / \mathrm{c} 3 \mathrm{tb} 20322 \mathrm{k}$
Magnetic NPs have gained much scientific interest for their unique magnetic properties such as superparamagnetism, high coercivity, low Curie temperature, and high magnetic susceptibility. ${ }^{\mathbf{1}, 2}$ Magnetic iron oxide nanoparticles (MIONs), owing to their advantages such as low toxicity and biocompatibility, are considered to be the most favourable candidate for bio-applications ${ }^{3}$ including magnetic resonance imaging (MRI), ${ }^{\mathbf{4}, 5}$ magnetic fluid hyperthermia (MFH), ${ }^{6}$ magnetic separation and immobilization of biomolecules such as nucleic acids and proteins, ${ }^{7}$ development of drug delivery systems for controlled release of drugs, ${ }^{8,9}$ biolabeling and magnetic sensors. ${ }^{10}$ Thermal decomposition and co-precipitation are among the most common techniques employed for MIONs preparation owing to their advantages but these are still associated with some limitations. ${ }^{2,11}$

Thermal decomposition of organo-metallic iron precursors at elevated temperatures in organic solvents and subsequent oxidation lead to highly monodisperse MIONs with high saturation magnetization because of separate nucleation and growth processes. ${ }^{12-14}$ However these NPs are not dispersible in 
water and thus cannot be directly used for bio-applications. For such applications they require laborious post-synthesis ligand exchange procedures ${ }^{\mathbf{1 5}, 16}$ which sometimes result in aggregation and loss of magnetic properties. ${ }^{17}$

Co-precipitation methods, however, result in the production of water-soluble magnetic NPs and are relatively cost-effective compared to the thermal decomposition methods. The magnetic NPs formed are, however, usually polydisperse and require cumbersome post-synthesis size selection procedures. $^{2,18}$ The polydispersity of the NPs is due to less control over complex hydrolysis reactions of the iron precursors and difficulty in the isolation of nucleation and growth steps. Furthermore, NPs obtained using co-precipitation methods often exhibit low crystallinity and low saturation magnetization due to low reaction temperature. ${ }^{19}$ Therefore it is still desired to develop simple, one step, cost effective and environmentally friendly protocols for the preparation of water-soluble, uniform, multifunctional, superparamagnetic iron oxide NPs with a better control over their size, shape and magnetic properties.

Multifunctional water-soluble polymers as capping ligands can play an important role in the preparation of MIONs and other inorganic NPs. ${ }^{20-22}$ In an aqueous co-precipitation process, polymer ligands can efficiently control the size and shape of the NPs due to the presence of abundant functional groups such as $-\mathrm{COOH},-\mathrm{OH},-\mathrm{NH}_{2}$ etc. These multifunctional polymers render magnetic NPs more stable, water-soluble and uniform in addition to providing them with rich surface chemistry which opens up ways for their easier post-synthesis modification and functionalization for bio-applications..$^{\mathbf{5 1 9 , 2 3 - 2 5}}$ Numerous studies have been reported for the synthesis of MIONs using polymer ligands in order to render them water-soluble and biocompatible. However, in most of the cases, the NPs are first prepared through thermal decomposition and then made water-soluble through post-synthesis modification processes such as ligand exchange using water-soluble polymer ligands. ${ }^{26}$

Previously we have reported the synthesis of water-soluble magnetic iron oxide NPs (MIONs, $D=4.5 \pm 0.4 \mathrm{~nm}$ ) using a multifunctional water-soluble polymer ligand (PTTM-PMAA) through a co-precipitation method..$^{19}$ It was found that the ratio between carboxylic acid groups present in the polymer ligand and iron precursors played an important role in determining the size and particle size distribution of the MIONs. Furthermore, by exploiting the free thiol $(-\mathrm{SH})$ and carboxylic acid $(-\mathrm{COOH})$ functional groups of the polymer ligand, postsynthesis modification of these NPs led to water-soluble fluorescent magnetic NPs through coupling with fluorescent dyes.

Recently, we have successfully described the application of these ultrasmall MIONs as efficient positive and negative dual contrast agents in magnetic resonance imaging (MRI). ${ }^{5}$ The in vitro and in vivo results demonstrated their better performance over clinically used gadolinium based $T_{1}$-positive (Gd-DTPA) and iron oxide based $T_{2}$-negative (SHU-555C) contrast agents. These MIONs have shown great potential as a better alternative to these single contrast agents especially to Gd-based $T_{1}$-positive contrast agents which have known clinical risks due to gadolinium toxicity. ${ }^{5}$

Herein we report a single step synthesis of highly watersoluble and ultrasmall $(4.6 \pm 0.7 \mathrm{~nm})$ MIONs using a new thioether end-functionalized polymer ligand dodecanethiolpolymethacrylic acid (DDT-PMAA). The effect of concentration and molecular weight of the polymer on the size and magnetic properties of the MIONs was also studied. A few modifications were made to our original high temperature single step coprecipitation method ${ }^{19}$ in order to improve the magnetic properties of MIONs, such as iron precursors were dissolved in concentrated $\mathrm{HCl}$ instead of $1 \mathrm{M} \mathrm{HCl}$ solution in order to prevent their hydrolysis and condensation before the addition of precipitating agents and the inert conditions were maintained, by nitrogen gas bubbling, throughout the course of reaction which not only protects MIONs from critical oxidation but also keeps their size smaller. ${ }^{27}$

MIONs prepared using DDT-PMAA have several carboxylic acid $(-\mathrm{COOH})$ functional groups which give them excellent solubility and stability in aqueous solutions. These MIONs showed high resistance against aggregation in aqueous media over a wide range of $\mathrm{pH}$ and salt concentrations due to excellent electrostatic and steric stabilization provided by the polymer ligand. These MIONs can be dried by evaporating the solvent and stored as powder for several months without any undesired changes in their chemical and physical properties. The polymer ligand offers MIONs better chemical stability against oxidation which otherwise leads to a decrease in their magnetic properties. Furthermore, cytotoxicity analysis of the MIONs proved them to be biocompatible even at high concentrations up to $500 \mu \mathrm{g} \mathrm{mL}{ }^{-1}$. The bio-applicability of these NPs was demonstrated by conjugating these MIONs with the anti-cancer drug doxorubicin and studying the efficacy of these drug-NP conjugates by incubating them with HepG2 cells, through MTT-assay. These drug-NP conjugates were found to have a better anti-cancer drug activity than that of the free drug. The improved drug activity in the case of drug-NP conjugates may be due to active NPs cellular uptake through endocytosis compared to the simple diffusion in the case of free drug. Finally, it was concluded that MIONs stabilized with DDT-PMAA are extremely biocompatible and can be used for several bio-applications due to their chemically rich surface providing numerous possibilities for their conjugation with a variety of therapeutic, targeting, and labelling agents.

\section{Experimental}

\subsection{Materials}

All chemicals were of analytical grade and used as-received without any further purification, unless otherwise described. Methacrylic acid (MAA, 99\%), 2,2'-azobisisobutyronitrile (AIBN, 98\%), anhydrous ethanol, anhydrous acetone and anhydrous diethyl ether were purchased from National Medicines Corporation Ltd. of P.R. China. Dodecanethiol (DDT, 99\%) was obtained from Aldrich. Ferric Chloride $\left(\mathrm{FeCl}_{3} \cdot 6 \mathrm{H}_{2} \mathrm{O}, 99 \%\right)$, ferrous sulfate $\left(\mathrm{FeSO}_{4} \cdot 7 \mathrm{H}_{2} \mathrm{O}, 99 \%\right)$, hydrochloric acid $(\mathrm{HCl}$, $38 \%)$ and ammonium hydroxide $\left(\mathrm{NH}_{4} \mathrm{OH}, 28 \%\right)$ were obtained from Sinopharm Chemical Reagent Co. Ltd. Doxorubicin hydrochloride was obtained from Pfizer Inc. $N$-(3-Dimethylamino-propyl)- $N$-ethylcarbodiimide hydrochloride (EDC) and $\mathrm{N}$-hydroxysuccinimide (NHS) were purchased from Aladdin Reagent Co., Ltd. (Shanghai, China). Fetal bovine serum (FBS) 
and Dulbecco's modified eagle's medium (DMEM) were obtained from Gibco (Basel, Switzerland). The 3-(4,5-dimethyltetrazolium bromide) (MTT) cell proliferation assay kit was received from Amresco (USA). Milli-Q water was used in the preparation and subsequent application experiments.

\subsection{Synthesis and characterization of the polymer ligand}

DDT-PMAA was synthesized by the chain transfer method using thiol as a chain transfer agent as described in previous reports. ${ }^{28,29}$ The molar ratio of the monomer to the chain transfer agent was altered in order to prepare four different molecular weight polymer samples. Detailed polymer ligand synthesis procedure and characterization results are provided in the ESI. $\dagger^{1} \mathrm{H}$ NMR spectra were recorded on a $400 \mathrm{MHz}$ Bruker AV400 spectrometer using $\mathrm{d}_{6}$-DMSO as a solvent in a $5 \mathrm{~mm}$ quartz NMR tube at room temperature using the $\delta$ scale and were consistent with the previous reports. ${ }^{28,29}$

\subsection{Preparation of MIONs}

The MIONs synthesis process was adopted from our previous work with little modification. ${ }^{19}$ A typical procedure involves the co-precipitation of an aqueous iron precursor solution with ammonia in the presence of a polymer ligand at high temperature (Scheme 1). Brief experimental details of the MIONs synthesis using the polymer ligand DDT-PMAA are provided in the ESI under Section ESI-3. $\dagger$

Twenty different samples of MIONs were prepared in the same way but with five different concentrations $(0.25,0.5,1,1.5$ and $2 \mathrm{mM}$ ) of four different polymer ligands (0.5\% DDT-PMAA, 2\% DDT-PMAA, 5\% DDT-PMAA and 10\% DDT-PMAA having molecular weights $14624,5330,3123$ and $2282 \mathrm{~g} \mathrm{~mol}^{-1}$ respectively), in order to obtain MIONs with optimum size and magnetic properties.

\subsection{Characterization of MIONs}

The particle size of the MIONs was determined by a high performance particle sizer (Brookhaven Nano DLS 90Plus/BIMAS) with multi-angle particle sizing option (from Brookhaven Instruments Co. USA) with an effective detection capability of 0.6 to $6000 \mathrm{~nm}$. The zeta potential values were determined by a Brookhaven ZetaPlus (Brookhaven Instruments Co. USA) at $25^{\circ} \mathrm{C}$ using the folded capillary cells. Data were obtained using a monomodal acquisition and was fit according to the Smoluchowski theory. After filtration with an aqueous membrane $(\Phi=13 \mathrm{~mm}, 0.22 \mu \mathrm{m})$, the samples were analyzed using

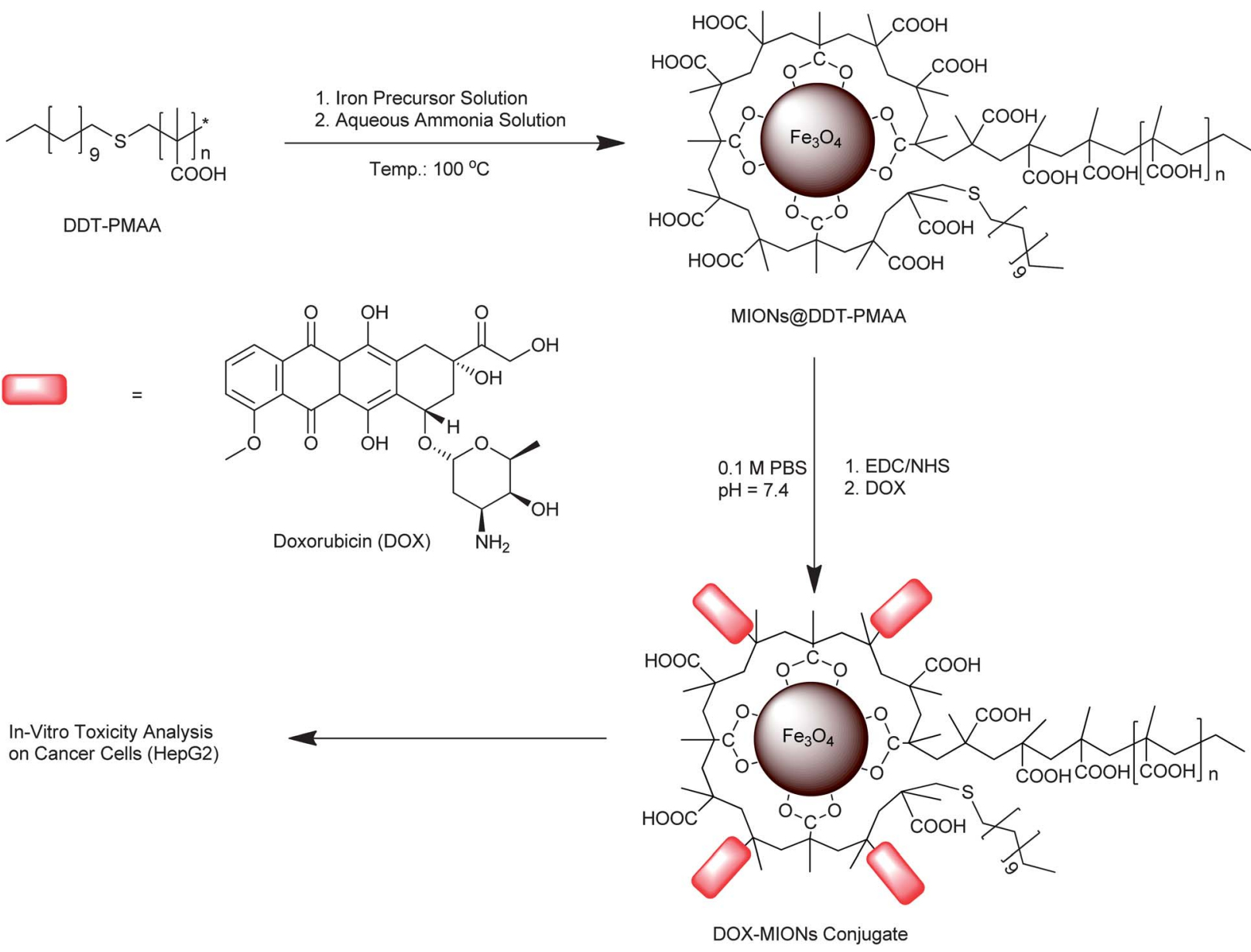

Scheme 1 Graphical representation of the synthesis of DDT-PMAA functionalized MIONs and their subsequent conjugation with DOX. 
ultrapure water as a solvent $(\mathrm{pH}=7)$. These measurements were run at least three times with independent particle batches. Transmission electron microscopy images were recorded on a JEOL-2100 electron microscope operating at an acceleration voltage of $200 \mathrm{kV}$. TEM samples were prepared by the slow evaporation of a drop of dilute aqueous solution of MIONs onto carbon-coated copper grids (400 mesh). The images were recorded with a Gatan 794 CCD camera. Size distribution graphs were prepared by analyzing about 200 individual NPs on each TEM image by ImageJ software. X-ray diffraction (XRD) was recorded on an X'Pert PRO XRD spectrometer (PANalytical B.V., Holland) using $\mathrm{Cu} \mathrm{K} \alpha(\lambda=1.54056 \AA)$ radiation in the $2 \theta$ range of $10-80^{\circ}$.

The UV-vis spectra were recorded using a Lambda 35 UV-vis spectrophotometer (Perkin-Elmer, U.S). The infrared spectra were recorded by a Fourier transform infrared (FTIR) spectrometer (Bruker Vertex 70) equipped with an attenuated total reflection (ATR) accessory. Raman spectra were recorded on a HR JOBIN YVON spectrometer using a laser of $632 \mathrm{~nm}$ and a $25 \%$ filter. Thermogravimetric analysis (TGA) measurements were performed using a TGA Q500 at a heating rate of $10{ }^{\circ} \mathrm{C}$ $\min ^{-1}$ from room temperature to $900{ }^{\circ} \mathrm{C}$ in an oxygen free atmosphere. The saturation magnetizations $\left(M_{\mathrm{S}}\right)$ of the MIONs were measured at $300 \mathrm{~K}$ on a Lakeshore 7400 Series vibrating sample magnetometer (VSM). All the magnetization data were normalized to the same weight.

\subsection{Toxicity analysis of MIONs}

Toxicity analysis of the MIONs was carried out before their bioapplication by MTT-assay using rat macrophage and HepG2 cells. The details of cell culturing and MTT-assay are provided in the ESI under Section SI- $6 . \dagger$

\subsection{Bio-applications of MIONs}

2.6.1 Synthesis of covalent conjugates (DOX-MIONs). DOX was covalently linked to MIONs via the formation of an amide bond between the carboxyl groups at the surface of MIONs and the amino groups of DOX through EDC coupling reaction. Briefly, the carboxyl groups of MIONs were first activated by NHS and EDC in the PBS (0.1 M, pH 7.4) solution at room temperature. DOX was then added and the $\mathrm{pH}$ of the reaction mixture was adjusted to 8.0 with dilute aqueous $\mathrm{NaOH}$ solution. The reaction was continued for $24 \mathrm{~h}$ in the dark with magnetic stirring. Then, excess reactants were removed by extensive dialysis against deionized water. The DOX conjugated MIONs were obtained by lyophilization and stored at $4{ }^{\circ} \mathrm{C}$ for further use.

2.6.2 Synthesis of electrostatic conjugates (DOX/MIONs). The non-covalent or electrostatic loading of DOX to MIONs was achieved through the electrostatic interaction between the carboxyl group of MIONs and the amine group of DOX. Specifically, MIONs and DOX were mixed in water at appropriate $\mathrm{pH}$ under stirring for $12 \mathrm{~h}$ in the dark. The DOX/MIONs was purified with magnetic separation and subjected to lyophilization to obtain DOX/MIONs powder.

2.6.3 Determination of drug loading efficiency. In order to determine the drug loading efficiencies of the drug-NP conjugates, a series of standard solutions of DOX was prepared in buffer $(\mathrm{pH}=7.4)$ and a standard curve was plotted between the concentration (along $X$-axis) and absorbance (along $Y$-axis) with the help of a UV-visible spectroscope at $\lambda_{\max }=480 \mathrm{~nm}$. Drug-NP conjugates were magnetically separated from the reaction mixture and were redispersed in the buffer again. The DOX concentration in supernatant fluids as well as in redispersed conjugate solutions was determined from the standard curve equation and drug loading efficiencies of drug-NP conjugates were calculated according to the following equation:

drug loading efficiency $(\%)=D_{\mathrm{IC}} / D_{\mathrm{LC}} \times 100$

where $D_{\text {IC }}$ is the initial concentration of DOX applied while $D_{\text {LC }}$ is the concentration of loaded DOX on drug-NP conjugates.

2.6.4 Cytotoxicity analysis of drug-NP conjugates. HepG2 (human hepatoblastoma) cells are usually used for cancer and apoptosis studies. In this study, HepG2 cells were used as the model cancer cell line in order to demonstrate the cytotoxicity of drug and MION conjugates. HepG2 cells were cultured in DMEM supplemented with 10\% FBS, streptomycin $(100 \mu \mathrm{g}$ $\left.\mathrm{mL}^{-1}\right)$ and penicillin $\left(100 \mathrm{U} \mathrm{mL}^{-1}\right)$. The cells were seeded at a density of $1 \times 10^{4}$ cells per well in $200 \mu \mathrm{L}$ of DMEM containing $10 \%$ FBS in 96-well microtiter plates and incubated for $24 \mathrm{~h}$. Media were replaced by fresh serum free DMEM media $(180 \mu \mathrm{L})$, and drug and drug-NP conjugate solutions were added to the culture media with final and equivalent DOX concentrations ( 5 , $10,25,50$ and $100 \mu \mathrm{g} \mathrm{mL}{ }^{-1}$ ). After $24 \mathrm{~h}$ of incubation, culture media were removed, and the cells were rinsed with PBS. The cells were then incubated with MTT $\left(0.5 \mathrm{mg} \mathrm{mL}^{-1}\right.$ in $200 \mu \mathrm{L}$ of supplemented serum free DMEM) at $37^{\circ} \mathrm{C}$ for $4 \mathrm{~h}$. Thereafter, the media were discarded and $150 \mu \mathrm{L}$ of DMSO was added to each well, and the plates were shaken for $15 \mathrm{~min}$ on a horizontal shaking bath at $37^{\circ} \mathrm{C}$. The absorbance was measured at $490 \mathrm{~nm}$. The sample-untreated cells in the medium were used as a positive reference. The cell viability (\%) was calculated according to the following equation:

$$
\text { cell viability }(\%)=\mathrm{OD}_{490}(\text { sample }) / \mathrm{OD}_{490}(\text { control }) \times 100
$$

MTT-assay was performed in order to determine the cytotoxicity of the drug-NP conjugates. Similarly, MTT-assays were performed after 48 and $72 \mathrm{~h}$ to determine the effect of incubation time period on activity of the drug-NP conjugates speculated indirectly from cell viability. ${ }^{30}$

\section{Results and discussion}

\subsection{Synthesis and characterization of the polymer ligand}

A series of polymer ligand DDT-PMAA, with four different molecular weights, was synthesized using dodecanethiol as a chain transfer agent by free radical polymerization of the monomer methacrylic acid (MAA) as described previously (Scheme S1 $\dagger$ ). GPC elution curves of four different polymer samples are shown in Fig. S1† and their molecular weights are given in Table S1.† 


\subsection{Preparation and characterization of polymer stabilized MIONs}

MIONs were synthesized by co-precipitation of an aqueous iron precursor solution containing $\mathrm{Fe}^{3+}$ and $\mathrm{Fe}^{2+}$ (molar ratio $2: 1$ ) with ammonia in the presence of DDT-PMAA. The size, shape and magnetic properties of the MIONs were controlled, by using different molecular weights and concentrations of the polymer ligand, during their preparation. This polymer ligand has already been proven as a good capping ligand for the synthesis of cobalt and gold NPs by some of us. ${ }^{23,28,29}$

It is known that rapid injection of precursors results in super saturation of chemical species in the reaction mixture which leads to an initial burst of nucleation at once followed by the growth of nuclei leading to the formation of monodisperse inorganic NPs. Whereas, slow drop-wise addition of precursors results in a continuous nucleation and growth processes in parallel resulting in a broad size distribution of NPs. ${ }^{12,31,32}$ However, in the case of magnetite NPs, the process is more complicated and difficult to control the nucleation and growth processes as iron precursors are initially hydrolyzed under alkaline conditions and then are condensed into iron oxide. Therefore, we dissolved iron precursors in concentrated $\mathrm{HCl}$ $(38 \%)$ in order to avoid their hydrolysis and condensation prior to the addition of precipitating agents. ${ }^{5}$ Both iron precursor solution and ammonia were rapidly added into the boiling aqueous solution of the polymer under a nitrogen atmosphere with vigorous stirring in order to keep nucleation and growth processes separate. Rapid injection of the precursor is widely used for uniform inorganic NPs synthesis in the organic phase by a thermal decomposition method..$^{12-14,33}$ The preparation of ultrasmall and uniform magnetic NPs with high crystallinity and magnetization through the usual co-precipitation method at room temperature is a tedious job, therefore we have carried out a co-precipitation process at high temperature $\left(100{ }^{\circ} \mathrm{C}\right),{ }^{19}$ which increases the reaction rate due to increased diffusion of active species and thus results in the formation of NPs with a narrow size distribution. ${ }^{19}$

The polymer ligand (DDT-PMAA) molecules cap the newly formed NPs through an interaction between carboxylic acid groups and the iron atoms on the NPs surface and thus restrict their further growth by compensating the surface energy due to electrostatic and steric stabilization which yields uniform as well as highly stable NPs. We have already proved this hypothesis by taking samples at different time intervals showing little further growth of NPs with the passage of time. ${ }^{19}$ It was also noticed that the use of a different ferrous precursor $\left(\mathrm{FeCl}_{2} \cdot 4 \mathrm{H}_{2} \mathrm{O}\right.$ instead of $\left.\mathrm{FeSO}_{4} \cdot 7 \mathrm{H}_{2} \mathrm{O}\right)$ had no effect on the shape, size and size distribution of the MIONs. Moreover, in comparison to the thermal decomposition method, this method involves no use of expensive and toxic organic precursors or solvents but reduces the reaction time and temperature; therefore it is more economical and eco-friendly.

The size of the magnetic NPs has two counter effects on their properties and scope of applications. The MIONs with ultrasmall size (5-20 nm) are easy to disperse in solutions and show higher stability against aggregation, however, they have very small magnetic moments and therefore are unable to be manipulated by an external magnetic field and thus cannot be used efficiently in certain applications such as magnetic separations but still can be good candidates for other applications like contrast agents for MRI. ${ }^{34}$ On the other hand, as the size of magnetic NPs increases their magnetization is improved due to their increased magnetic moments which make them more attractive for magnetic separation applications; however, they have poor dispersion capability and usually undergo aggregation. Thus, for any particular application, it is necessary to prepare magnetic NPs with desired size and magnetic properties. $^{23}$

In this work, dried magnetic NPs prepared with DDT-PMAA were re-dispersed in Milli-Q water to make aqueous dispersions and without any size selection procedure hydrodynamic sizes of magnetic NPs were determined by dynamic light scattering (DLS) which are shown in (Fig. 1). It was observed from DLS analysis that as the molecular weight of the polymer ligand is gradually decreased, the size of the MIONs is steadily increased (Fig. 1a-d). It may be attributed to the inefficient capping of growing iron oxide nuclei by smaller polymer molecules (Fig. 1d). However, using four different molecular weight polymers, the size of the NPs was initially decreased with an increase in the concentration of the polymer ligand but then the size of NPs was increased again. This may be due to the better diffusion of all these polymers at lower, but still enough, concentration to approach the surface of growing nuclei of iron oxide thus capping them at the earlier stage and limiting their growth. At higher polymer concentration, the diffusion of chemical species is restricted due to high viscosity of the solution and thus allowing the iron oxide nuclei to grow into larger NPs. ${ }^{19}$

In order to determine the size of MIONs, transmission electron microscopy (TEM) was also performed for selected samples which showed smaller diameter compared to that obtained by DLS analysis. TEM analysis showed that MIONs
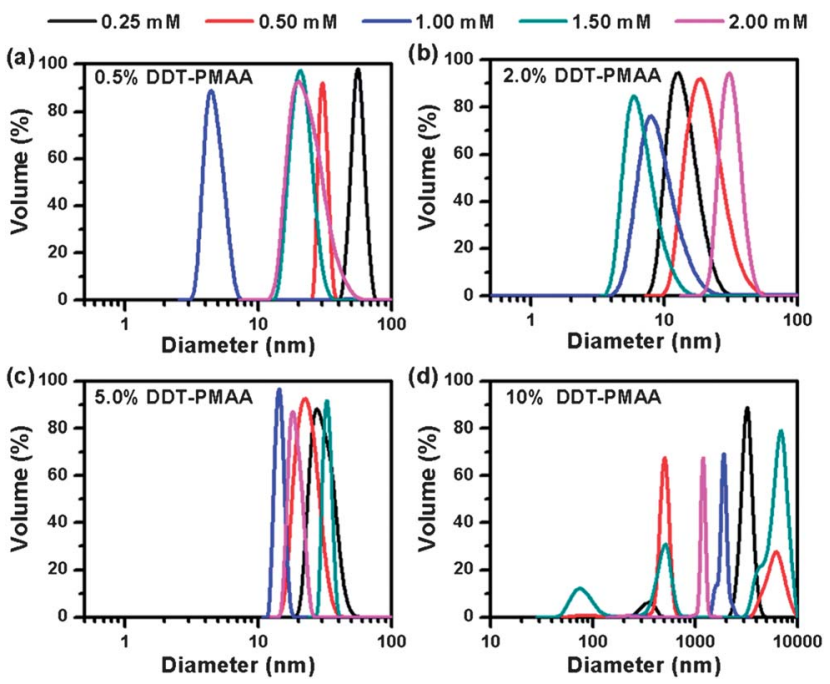

Fig. 1 DLS curves of MIONs prepared with different concentrations of (a) $0.5 \%$ DDT-PMAA (b) 2\% DDT-PMAA (c) 5\% DDT-PMAA and (d) 10\% DDT-PMAA 


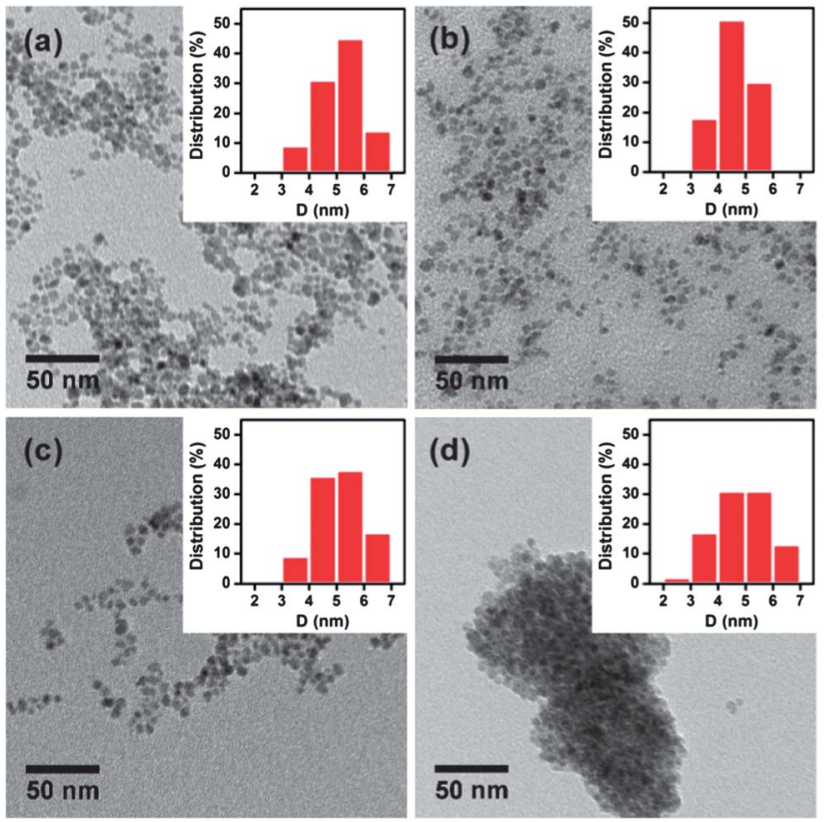

Fig. 2 (a) TEM images along with particle size distribution histograms of MIONs prepared with $1.5 \mathrm{mM}$ of $0.5 \%$ DDT-PMAA (a), 2\% DDT-PMAA (b), 5\% DDTPMAA (c) and 10\% DDT-PMAA (d).

prepared with $1.5 \mathrm{mM}$ 0.5\% DDT-PMAA $\left(M_{\mathrm{n}}=14624 \mathrm{~g} \mathrm{~mol}^{-1}\right)$ were in the size range of $c a .5 .1 \pm 0.8 \mathrm{~nm}$ (Fig. 2a) which is much smaller than their hydrodynamic diameter obtained from DLS (Fig. 1a). In the case of MIONs prepared with $1.5 \mathrm{mM} 2 \%$ DDTPMAA $\left(M_{\mathrm{n}}=5330 \mathrm{~g} \mathrm{~mol}{ }^{-1}\right)$, the hydrodynamic diameter obtained from DLS was $5.9 \mathrm{~nm}$ (Fig. 1b) while its diameter obtained from TEM was found to be $4.6 \pm 0.7 \mathrm{~nm}$ (Fig. 2b). It is noteworthy that the actual size of the MIONs obtained with TEM was much smaller than the hydrodynamic radii obtained from the DLS method, which can be attributed to the presence of the extended layer of the hydrated polymer ligand around these NPs when dispersed in aqueous media and was also observed by Sun and co-workers. ${ }^{35}$ Similarly, MIONs prepared with $1.5 \mathrm{mM} \mathrm{5 \%}$ DDT-PMAA (3123 $\mathrm{g} \mathrm{mol}^{-1}$ ) had a hydrodynamic diameter of $30 \mathrm{~nm}$ (Fig. 1c) but the actual diameter obtained from TEM was found to be $5.0 \pm 1.0 \mathrm{~nm}$ (Fig. 2c). As the molecular weight of the polymer is further decreased to $2281 \mathrm{~g} \mathrm{~mol}^{-1}$, in the case of $10 \%$ DDT-PMAA, an intense increase in the size of NPs (over $100 \mathrm{~nm}$ ) was observed by DLS analysis (Fig. 1d). It was interesting to note that the increase in polymer concentration did not help in reducing the size of MIONs obtained with $10 \%$ DDTPMAA (Fig. 1d). However, TEM analysis of these MIONs obtained with 10\% DDT-PMAA (1.5 mM) (Fig. 2d) revealed that this increase in size is due to massive agglomeration of the smaller NPs i.e., 4.7 to $5.9 \mathrm{~nm}$. MIONs obtained with $10 \%$ DDTPMAA were not very well dispersed in aqueous solution and were settled down within a few days due to their large agglomeration rate and thus settling down under the force of gravity. These results revealed that in addition to the concentration of the polymer ligand (carboxylic acid groups to iron precursor ratio), the molecular weight or in other words the length of the polymer molecular chain is another important and critical factor in determining the size and size-distribution of the MIONs. TEM images and size distribution histograms of all the MION samples prepared with different concentrations and molecular weights of DDT-PMAA are provided in the ESI (Fig. S2-S5†).

In order to study the surface chemistry of MIONs, they were characterized by Fourier transform infrared spectroscopy (FTIR). The comparison of FTIR spectra of 2\% DDT-PMAA, MIONs prepared with (1.5 mM) 2\% DDT-PMAA and bare MIONs (without any capping ligand) is shown in Fig. S6. $\dagger$ The FTIR spectrum of the polymer ligand has a strong absorption peak at $1704 \mathrm{~cm}^{-1}$ corresponding to the asymmetric stretching of the carbonyl (-CO-) group of the polymer ligand, which is extensively reduced in the spectrum of MIONs with polymer which indicates that the polymer is bound to the surface of iron oxide NPs. ${ }^{36}$ Additionally a broad peak at $3124 \mathrm{~cm}^{-1}$ is attributed to the O-H stretching of carboxylic acid groups and is observed in FTIR spectra of DDT-PMAA and MIONs@DDT-PMAA but completely absent in the case of bare MIONs. ${ }^{28,37}$ Furthermore, the asymmetric and symmetric stretching bands of carboxylate (-COO-) present in the pure polymer ligand spectrum are shifted from 1485 and 1390 to 1556 and $1402 \mathrm{~cm}^{-1}$ respectively in the spectrum of MIONs protected with a polymer ligand which confirms the presence of a polymer ligand on the NPs surface. Finally, the characteristic peaks appearing at 592 and $448 \mathrm{~cm}^{-1}$ are assigned to the torsion vibration and stretching mode of the $\mathrm{Fe}-\mathrm{O}$ bond of magnetite present only in FTIR spectra of MIONs with DDT-PMAA and bare MIONs. ${ }^{19,38}$

The crystalline structure of the MIONs was determined by XRD measurement as shown in Fig. S7†. It can be clearly seen that the peaks present at $2 \theta=30.1,35.5,43.3,53.4,57.3$ and $62.7^{\circ}$ corresponding to (220), (311), (400), (422), (511) and (440) reflections of magnetite, respectively indicate the presence of the crystalline spinel structured magnetite $\left(\mathrm{Fe}_{3} \mathrm{O}_{4}\right)$ phase of iron oxide. ${ }^{39}$ The wide peak at $2 \theta=18.7^{\circ}$ is assigned to the polymer which was also found in the XRD pattern of the neat polymer (data not shown). ${ }^{8}$ However, the phase of the iron oxide in magnetic NPs cannot be confirmed by simple FTIR and XRD because they are less sensitive to it. ${ }^{19}$ Therefore Raman spectra of the magnetic NPs prepared in the presence/absence of nitrogen were recorded (Fig. S8 $\dagger$ ). A characteristic peak of magnetite at $665 \mathrm{~cm}^{-1}$ is present in the Raman spectrum (Fig. S8b $\dagger$ ) which is significantly reduced in intensity in the case of oxidized iron oxide NPs indicating the major iron oxide phase to be $\mathrm{Fe}_{3} \mathrm{O}_{4}{ }^{40}$ Furthermore, the peaks present at 384,471 , and $587 \mathrm{~cm}^{-1}$ in the Raman spectrum (Fig. S8a†) are characteristic peaks for maghemite $\left(\gamma-\mathrm{Fe}_{2} \mathrm{O}_{3}\right)$ which are absent in the magnetite Raman spectrum. The formation of stable hematite $\left(\alpha-\mathrm{Fe}_{2} \mathrm{O}_{3}\right)$ from maghemite $\left(\gamma-\mathrm{Fe}_{2} \mathrm{O}_{3}\right)$ by oxidation is indicated by the appearance of strong peaks at 209 and $268 \mathrm{~cm}^{-1}$ in the maghemite Raman spectrum (Fig. S8a †). ${ }^{\mathbf{1 9 4 0 - 4 2}}$

To further investigate the thermal stability of the MIONs, and to deduce the iron oxide and polymer contents of the NPs, thermogravimetric analysis was performed for 2\% DDT-PMAA, MIONs with 2\% DDT-PMAA (1.5 mM) and bare MIONs. The neat polymer ligand ( $2 \%$ DDT-PMAA) was almost completely decomposed at $540{ }^{\circ} \mathrm{C}$ with almost no residue left. From TGA 
analysis, we concluded that the polymer wt $\%$ in the MIONs with $2 \%$ DDT-PMAA (1.5 mM) was $70 \%$ and iron oxide wt $\%$ was $30 \%$ (Fig. S9†).

Saturation magnetization of the selected samples of MIONs prepared with different DDT-PMAA ligands was determined by a Vibrating Sample Magnetometer (VSM) at room temperature. In order to calculate the exact magnetization of these MIONs, the effect of magnetically dead polymer layer was excluded and then compared with magnetization of bare MIONs without the polymer (Fig. S10†). The corrected magnetization of the MIONs with $2 \%$ DDT-PMAA (1.5 mM) having a TEM diameter about 4.6 $\mathrm{nm}$ was found to be $50 \mathrm{emu}^{-1}$ which is comparable to the literature for MIONs of comparable dimensions. ${ }^{19}$ The low saturation magnetization of $4.6 \mathrm{~nm}$ MIONs compared to the bare MIONs is due to their ultra-small size as saturation magnetization of magnetic NPs is directly related to their size. ${ }^{43}$

Dried MIONs prepared with 0.5, 2 and 5\% DDT-PMAA were highly stable and readily re-dispersible in water. $\mathrm{pH}$ and salt stability tests were performed with aqueous dispersions of MIONs prepared with 2\% DDT-PMAA $(1.5 \mathrm{mM})$ and were found to be stable within a wide range of $\mathrm{pH}(05-10)$ and up to high concentration of salt $(\mathrm{NaCl})$ concentration $(1 \mathrm{M})$ (Fig. S11†). High stability of the MION dispersions can be attributed to the large negative zeta potential and excellent electrostatic as well as steric stabilization by the polymer ligand owing to the large abundance of carboxylic acid groups.

\subsection{Toxicity analysis and bioapplication of MIONs}

In vitro cytotoxicity tests of the MIONs and polymer ligands demonstrated that both polymer and polymer stabilized MIONs were non-toxic to the cells even when used up to very high concentration $\left(1000 \mu \mathrm{g} \mathrm{mL} \mathrm{m}^{-1}\right)$.

However the MIONs without any polymer ligands showed significant toxicity towards cells, and reduced the cell viability in a time dependent and dose dependent manner when their concentration was exceeded beyond $25 \mu \mathrm{g} \mathrm{mL} \mathrm{m}^{-1}$ (Fig. 3 and $\mathrm{S} 12 \dagger)$. This may be due to the fact that MIONs without polymer ligand are quite large in size and more irregular in shape compared to MIONs@DDT-PMAA which may be responsible for cellular toxicity. Moreover, bare MIONs act as a source of ferrous ions and highly reactive hydroxyl radicals which are toxic to the cells. ${ }^{44}$

With the increase in concentration of these bare MIONs, cell viability was further reduced even more promptly for a longer exposure duration and at MION concentrations of 500 and 1000 $\mu \mathrm{g} \mathrm{mL}{ }^{-1}$, and cell survival rates were lower than 50\% (Fig. S12 $\dagger$ ). On the other hand, the polymer ligand itself and MIONs capped with the polymer ligand are totally biocompatible due to the biocompatible nature of the polymer ligand (DDT-PMAA). The optical micrographs of the cells for $24 \mathrm{~h}$ incubation time (Fig. 4) show almost no decrease in cell density with the increasing concentration of DDT-PMAA and MIONs@DDT-PMAA (Fig. 4, top and middle rows respectively). While a noticeable decrease in cell density can be seen with an increase in concentration of bare MIONs (Fig. 4, bottom row). Bare MIONs have a large size, broad size distribution $(10 \pm 3 \mathrm{~nm})$ and irregular shape

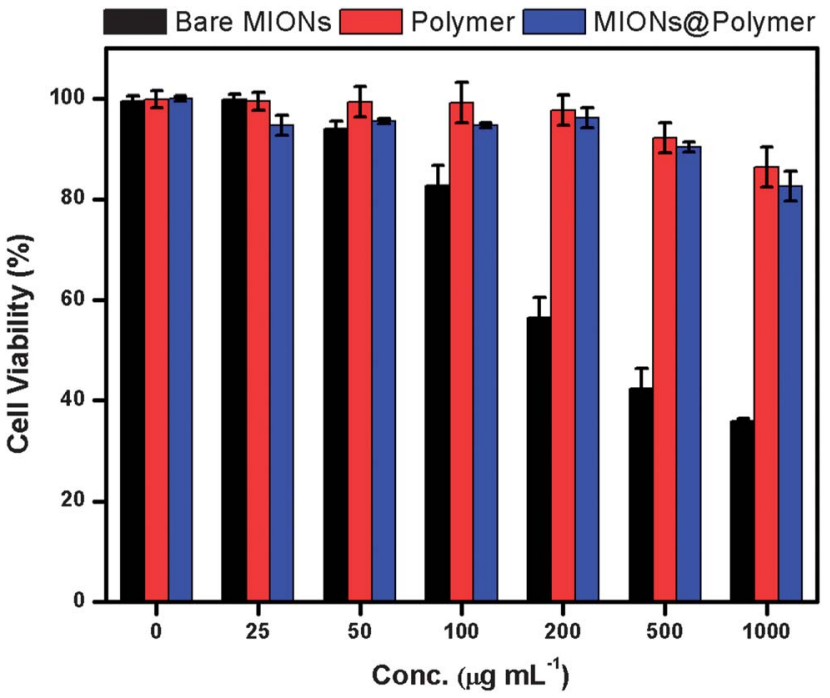

Fig. 3 Cell viability of rat macrophages determined by MTT-assay after $24 \mathrm{~h}$ incubation with various concentrations of bare MIONs, DDT-PMAA and MIONs@DDT-PMAA.

(Fig. S13†). Since they are not stabilized with any capping ligand, they tend to aggregate and gather around the cells posing oxidative and mechanical stress to the cells and restricting their growth. When the concentrations of bare MIONs are very high $\left(200-1000 \mu \mathrm{g} \mathrm{mL}{ }^{-1}\right)$, cell viability is reduced up to $30 \%$ and the cells are heavily surrounded by NP aggregates which makes it difficult to distinguish between the cells and MION aggregates (Fig. 4, bottom row). These results confirm that, in contrast to bare MIONs, the polymer ligand and MIONs capped with the polymer ligand are non-toxic and very biocompatible. Similarly, the cell viability of HepG2 was also determined by MTT-assay after 24, 48 and $72 \mathrm{~h}$ of incubation with MIONs@2\% DDT-PMAA and they were found to be nontoxic to HepG2 even up to high concentrations (Fig. S14†).

MIONs prepared with DDT-PMAA have special characteristics such as high water solubility and large negative zeta potential and excellent stability in a wide range of $\mathrm{pH}$ and salt concentrations, which make them highly suitable for bioapplications such as contrast agents for MRI, magnetic separation of biomolecules and cells, and development of NP based drug delivery systems. Furthermore, the presence of abundant carboxylic acid groups $(-\mathrm{COOH})$ on the MIONs surface also provide great opportunities to simultaneously modify these NPs with targeting, tracing and therapeutic agents for multipurpose bio-probes.

In order to explore the applications of MION@DDT-PMAA in drug delivery, they were conjugated with doxorubicin (Scheme 1) and their zeta potential measurement indicated the presence of drug on MIONs. The zeta potential value of the MIONs before drug loading was as low as $-60 \mathrm{mV}$ because of abundant surface carboxylic acid groups of the polymer capping ligand (Fig. 5).

However, loading of DOX resulted in an increase in zeta potential of MIONs. In the case of covalent conjugation of the drug, some of the surface carboxylic acid groups on the MIONS undergo coupling reaction with amine groups of the drug 


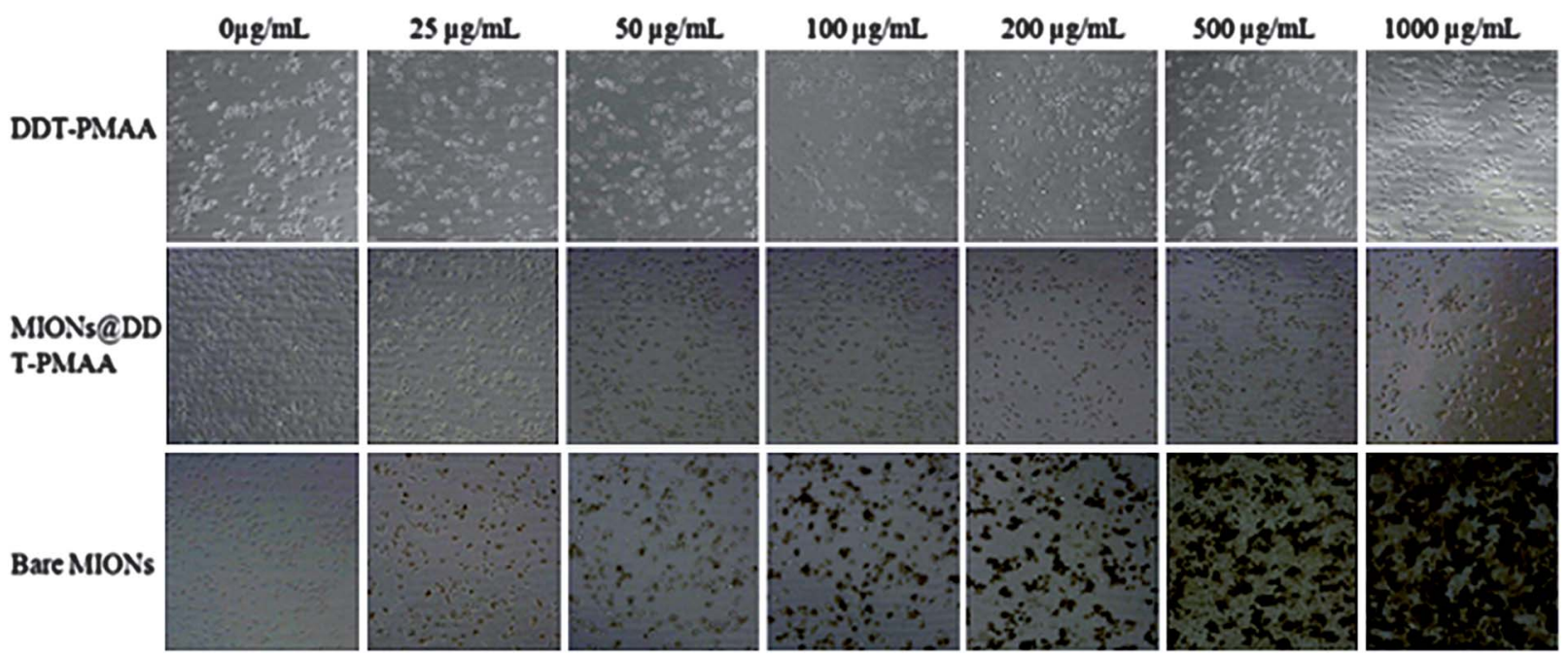

Fig. 4 Optical microscopic images of the cells growing with different concentrations of DDT-PMAA (top row), MIONs@DDT-PMAA (middle row) and bare MIONs (bottom row) taken after $24 \mathrm{~h}$ of the incubation time period.

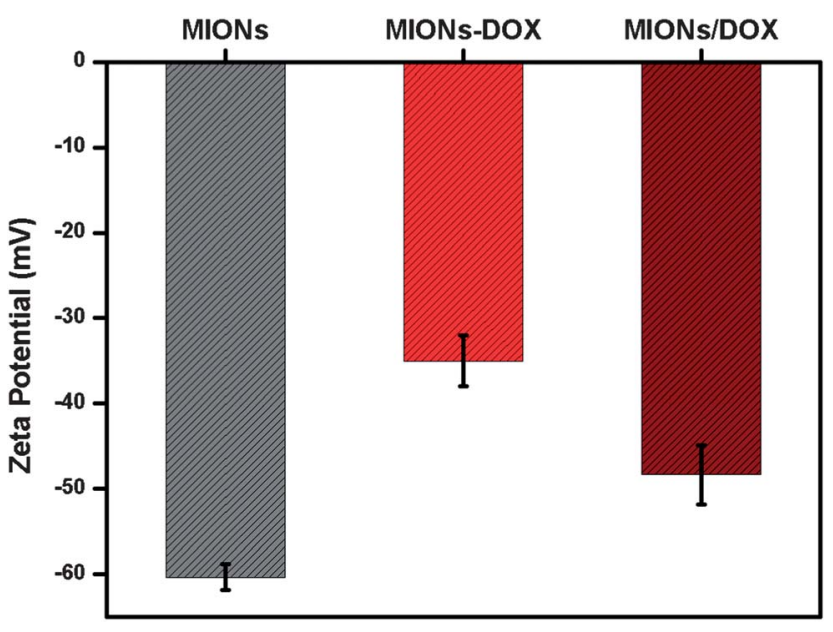

Fig. 5 Zeta potentials of MIONs, DOX-MIONs and DOX/MIONs in PBS (10 mM $\mathrm{pH}$ 7.4). Each result represents the mean of three runs with \pm S.D.

molecules resulting in an increase in zeta potential of the final conjugate $(-35 \mathrm{mV})$. However, in the case of electrostatic loading, the relatively small increase in the zeta potential $(-48$ $\mathrm{mV}$ ) indicates lower drug loading compared to that achieved by covalent coupling..$^{45}$

The conjugation of the DOX to the MIONs is also evidenced from the FTIR spectra of DOX-MIONs (Fig. 6). The FTIR spectrum of pure DOX shows peaks at 3456 and $3335 \mathrm{~cm}^{-1}$ which are due to $\mathrm{N}-\mathrm{H}$ stretching vibrations of the primary amine structure and $\mathrm{O}-\mathrm{H}$ stretching vibrations (Fig. 6a) respectively. Whereas in the case of DOX-conjugated polymer coated MIONs, these peaks are overlapped, broadened and are shifted to the lower frequency range $\left(3260 \mathrm{~cm}^{-1}\right) .{ }^{46}$ Furthermore, the peaks observed at 868 and $807 \mathrm{~cm}^{-1}$ due to $\mathrm{N}-\mathrm{H}$ wag in pure DOX were reduced in the FTIR spectrum of DOX-conjugated MIONs and a characteristic band of Fe-O was appeared (Fig. 6b). Similarly, the appearance of the other characteristic peaks of the pure DOX was also observed in the FTIR spectrum of the DOX-

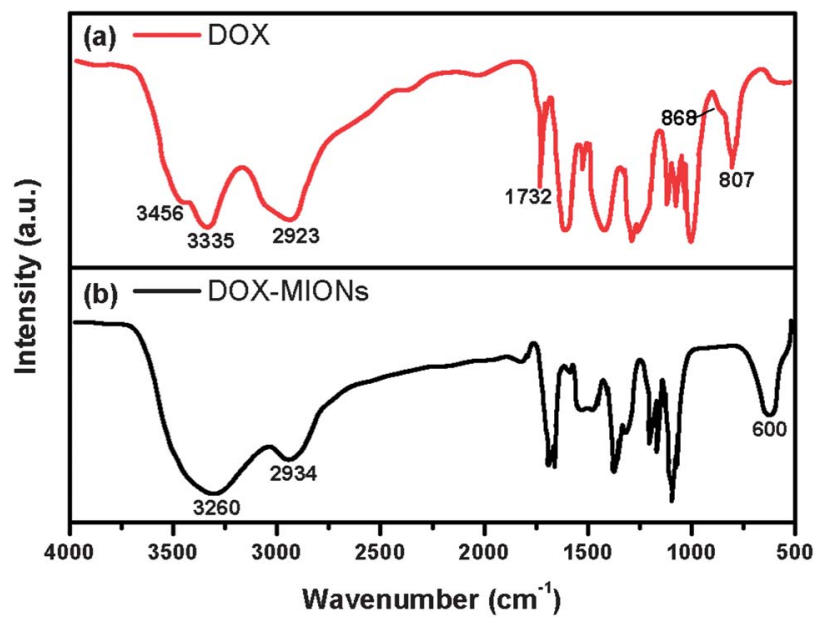

Fig. 6 FTIR spectra of pure DOX (a) and DOX-MIONs (b).

MIONs and collectively this evidence confirms the covalent conjugation of the drug to the MIONs.

UV-visible spectra of DOX, MIONs and their conjugates and a standard curve of DOX are presented in Fig. S15. $\dagger$ Drug-NP conjugates were magnetically separated from the reaction mixture as shown in Fig. S16 $\dagger$ and were redispersed in the buffer again. The DOX concentration in supernatant fluids as well as in redispersed conjugate solutions was determined from the standard UV-visible standard curve equation. Drug loading efficiencies of drug-NP conjugates were calculated to be $60 \%$ and $57 \%$ for covalent (DOX-MIONs) and (DOX/MIONs) electrostatic drug-NP conjugates respectively. TEM examination showed no significant change in size and size distribution of individual particles except a little aggregation (Fig. S17†).

The cytotoxicity of drug and NP conjugates was determined on HepG2 (human hepatoblastoma) cells through MTT-assay at different time intervals i.e. after 24,48 and $72 \mathrm{~h}$ as shown in Fig. 7. In vitro cytotoxicity of DOX and MIONs were indirectly 
speculated from cell viability in the presence of free DOX and its conjugates with MIONs. In these experiments, DOX is used as a positive control and the \% cell viability was calculated considering cell viability of the samples treated with no DOX as $100 \%$. It was observed that in all cases (free DOX, DOX-MIONs, and DOX/MIONs), the activity of the drug was found to be dose as well as time dependent. Cell viability of the HepG2 cells was gradually decreased with increase in the drug concentration ( $\mu \mathrm{g}$ $\mathrm{mL}^{-1}$ ). It is clearly evidenced that in all cases, cell viability of the HepG2 cells is decreased with an increased exposure to DOX in the cell medium either in the form of free DOX or its conjugate with MIONs. These results are logical and in consistent with the previous results. ${ }^{45}$

After $24 \mathrm{~h}$, the anti-cancer activity of free DOX was observed to be more prominent (Fig. 7a) i.e. cell viability was reduced to $50 \%$, which can be attributed to the higher cellular uptake of drug through simple diffusion, ${ }^{45}$ since it has been well established in the literature that the pure doxorubicin is taken up by the cells only through Fickian diffusion. ${ }^{47,48}$ However in the case

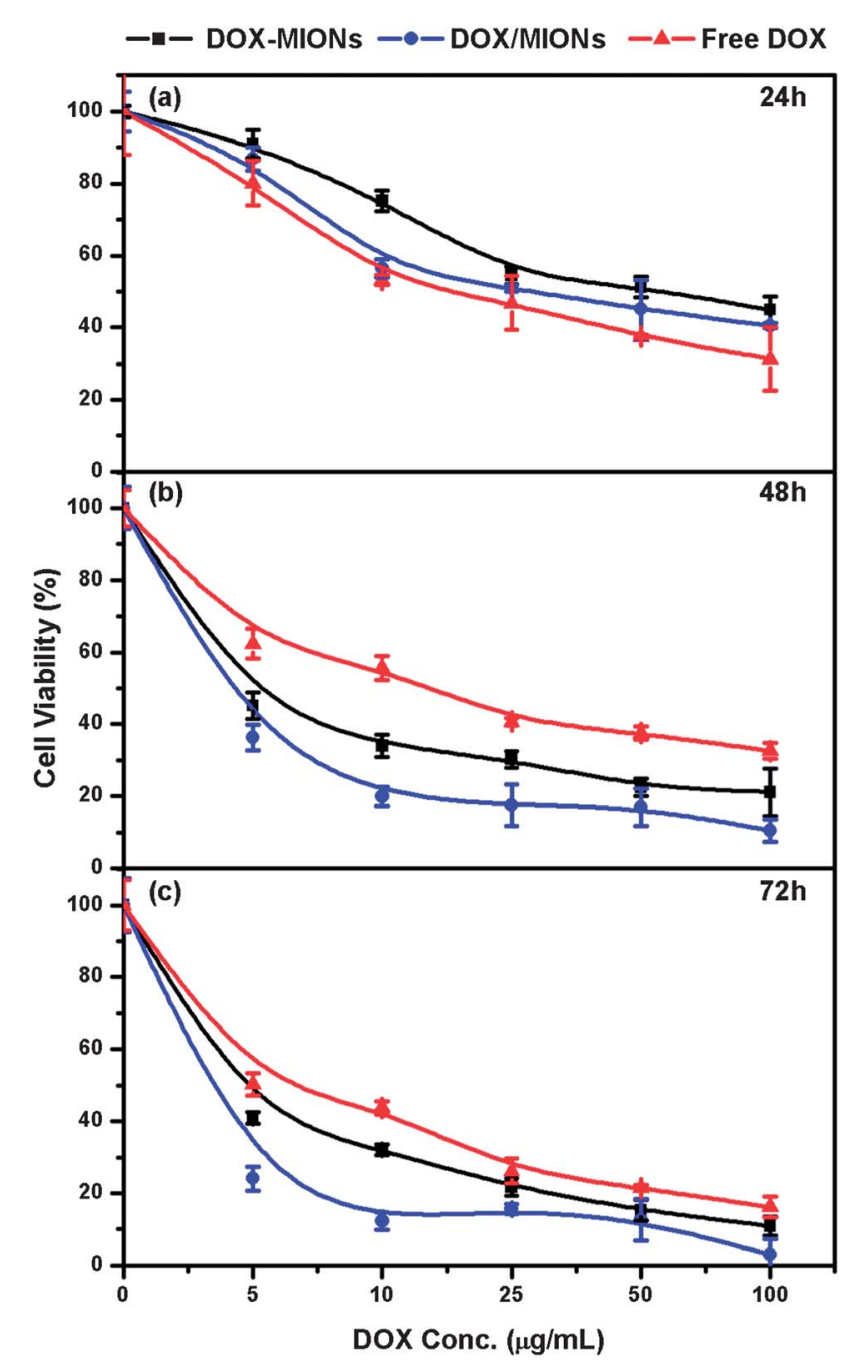

Fig. 7 Cell viability of HepG2 cells incubated with DOX-MIONs and DOX/MIONs and free DOX with an equivalent DOX concentration determined after (a) $24 \mathrm{~h}$, (b) $48 \mathrm{~h}$, and (c) $72 \mathrm{~h}$. Each data point represent the mean \pm S.D. of three experiments. of DOX-MIONs and DOX/MIONs, the cellular uptake of the drug was carried out more likely through endocytosis. It means that conjugation of the drug with NPs facilitated the cellular uptake of DOX through endocytosis which was probably not the case with the free drug. ${ }^{\mathbf{4 5 , 4 9}}$ Thus, after $24 \mathrm{~h}$, it was observed that free DOX showed higher activity while its covalent conjugates with MIONs showed reduced activity.

However, later on (in MTT-assays performed at 48 and $72 \mathrm{~h}$ of incubation time) free DOX had less cytotoxicity whereas DOX/ MIONs showed the maximum activity as shown in Fig. $7 \mathrm{~b}$ and $\mathrm{c}$. The higher activity of the DOX/MIONs may be attributed to the more efficient cellular uptake of drug at later stages through endocytosis as compared to the simple diffusion of free DOX. The higher cytotoxicity activity of the drug and NP conjugates (DOX-MIONs and DOX/MIONs) can also be attributed to the higher solubility of these conjugates at physiological temperature and $\mathrm{pH}$ as compared to that of the free DOX.

The anti-cancer activity of the DOX-MIONs was higher than that of free DOX but less than that of DOX/MIONs which is due to the fact that DOX is known to chelate with DNA inside the nucleus and arrest the cell functions causing cell death. Since, in DOX-MION conjugates, the DOX is linked covalently to the MIONs so probably it is not easily released inside the cytoplasm and thus its transport to the nucleus is restricted. However, in the case of DOX/MIONs, since the DOX is linked to the MIONs only through electrostatic interactions, once inside the cell, the DOX is easily released from conjugates under the chemical environment within the cytoplasm and is available to penetrate within the nucleus of the cell and chelate to DNA to arrest its functions and cause cell death. ${ }^{50}$

To summarize, this work demonstrates the synthesis of the highly water-soluble MIONs which can be easily conjugated to DOX covalently or electrostatically with high drug loading efficiency. The DOX/MIONs were observed to have higher cytotoxicity than DOX-MIONs and free DOX possibly due to their efficient cellular uptake through endocytosis compared to passive diffusion. The exact mechanism of cellular uptake of NPs is, however, a complex phenomenon and needs extensive exploration. From cytotoxicity analysis and the behaviour of DOX conjugated MIONs, it was observed that they have potential bio-applications owing to their excellent water solubility, high stability and biocompatibility. Moreover, they possess a chemically enriched surface for an easy conjugation with the biomolecules, biomarkers, and therapeutic agents of choice for their subsequent applications in MRI, multimodal imaging, and drug delivery.

\section{Conclusions}

Biocompatible ultra-small magnetic iron oxide $\left(\mathrm{Fe}_{3} \mathrm{O}_{4}\right)$ NPs were prepared by co-precipitation of an aqueous iron precursor solution at high temperature in the presence of a water-soluble thioether end-functionalized polymer ligand DDT-PMAA. These MIONs are fairly uniform in size and can be dried by solvent evaporation so that it could be stored for a long time. The dried powders of MIONs are readily re-dispersible in water without any significant change in their size and size 
distribution. MIONs aqueous dispersion was highly stable even at high salt concentrations and also over a wide range of $\mathrm{pH}$. The molecular weight and concentration of the polymer ligand showed a significant effect on the size and magnetic properties of MIONs.

MIONs were found to be highly biocompatible as determined by MTT-assay. The bio-applicability of MIONs was demonstrated by developing NP based anti-cancer drug conjugates. DOX was used as a model drug and its amine groups were conjugated to the carboxylic acid groups of MIONs through electrostatic and covalent interactions to prepare DOX/MIONs and DOX-MIONs respectively. NPs and drug conjugates showed high drug loading efficiency and demonstrated higher cytotoxicity towards HepG2 cancer cells due to their improved solubility and cellular uptake compared to the free drug. It was observed that the drug loading mode can significantly affect the drug efficiency and this study can help in the optimization of development of the MIONs based drug delivery formulations. Furthermore, MIONs stabilized with DDT-PMAA have rich surface chemistry which can be exploited for the conjugation of a variety of therapeutic, targeting, and labelling agents for several bio-analytical and drug delivery applications.

\section{Acknowledgements}

The authors thank the Analytical and Testing Center of Huazhong University of Science \& Technology for characterization assistance. This work was supported by the National Natural Science Foundation of China (No. 50973037, 51173058), the Program for New Century Excellent Talents in University from the Ministry of Education of China (No. NCET-10-0389), Natural Science Foundation of Hubei Province (2012FFB00203), and the Hubei Province Natural Science Fund for Distinguished Young Scholars (No. 2011CDA109).

\section{Notes and references}

1 Y. Tai, L. Wang, G. Yan, J.-m. Gao, H. Yu and L. Zhang, Polym. Int., 2011, 60, 976-994.

2 A.-H. Lu, E. L. Salabas and F. Schüth, Angew. Chem., Int. Ed., 2007, 46, 1222-1244.

3 J. Xie, J. Huang, X. Li, S. Sun and X. Chen, Curr. Med. Chem., 2009, 16, 1278-1294.

4 H. B. Na, I. C. Song and T. Hyeon, Adv. Mater., 2009, 21, 2133-2148.

5 Z. Li, P. W. Yi, Q. Sun, H. Lei, H. Li Zhao, Z. H. Zhu, S. C. Smith, M. B. Lan and G. Q. M. Lu, Adv. Funct. Mater., 2012, 22, 2387-2393.

6 S. Laurent, S. Dutz, U. O. Häfeli and M. Mahmoudi, Adv. Colloid Interface Sci., 2011, 166, 8-23.

7 B. Sun, L. Huang, N. Na, D. He and J. Ouyang, Electrophoresis, 2011, 32, 2091-2098.

8 L. Zhou, B. He and F. Zhang, ACS Appl. Mater. Interfaces, 2012, 4, 192-199.

9 F. Dilnawaz, A. Singh, C. Mohanty and S. K. Sahoo, Biomaterials, 2010, 31, 3694-3706.
10 J. B. Haun, T.-J. Yoon, H. Lee and R. Weissleder, Wiley Interdiscip. Rev.: Nanomed. Nanobiotechnol., 2010, 2, 291304.

11 Y. L. Tai, L. Wang, G. Q. Fan, J. M. Gao, H. J. Yu and L. Zhang, Polym. Int., 2011, 60, 976-994.

12 M. F. Casula, Y.-w. Jun, D. J. Zaziski, E. M. Chan, A. Corrias and A. P. Alivisatos, J. Am. Chem. Soc., 2006, 128, 1675-1682.

13 S. Sun and H. Zeng, J. Am. Chem. Soc., 2002, 124, 8204-8205.

14 J. Park, E. Lee, N.-M. Hwang, M. Kang, S. C. Kim, Y. Hwang, J.-G. Park, H.-J. Noh, J.-Y. Kim, J.-H. Park and T. Hyeon, Angew. Chem., Int. Ed., 2005, 44, 2872-2877.

15 H. Gu, K. Xu, C. Xu and B. Xu, Chem. Commun., 2006, 941.

16 Y.-M. Huh, Y.-w. Jun, H.-T. Song, S. Kim, J.-s. Choi, J.-H. Lee, S. Yoon, K.-S. Kim, J.-S. Shin, J.-S. Suh and J. Cheon, J. Am. Chem. Soc., 2005, 127, 12387-12391.

17 A. B. Bourlinos, A. Bakandritsos, V. Georgakilas and D. Petridis, Chem. Mater., 2002, 14, 3226-3228.

18 W. Wu, Q. He and C. Jiang, Nanoscale Res. Lett., 2008, 3, 397415.

19 Z. Li, B. Tan, M. Allix, A. I. Cooper and M. J. Rosseinsky, Small, 2008, 4, 231-239.

20 H. Xin, Z. Hui, L. Liyun and T. Bien, Progr. Chem., 2010, 22, 953-961.

21 R. B. Grubbs, Polym. Rev., 2007, 47, 197-215.

22 M. Chanana, Z. W. Mao and D. Y. Wang, J. Biomed. Nanotechnol., 2009, 5, 652-668.

23 L. T. Lu, L. D. Tung, I. Robinson, D. Ung, B. Tan, J. Long, A. I. Cooper, D. G. Fernig and N. T. K. Thanh, J. Mater. Chem., 2008, 18, 2453.

24 L. T. Lu, L. D. Tung, J. Long, D. G. Fernig and N. T. K. Thanh, J. Mater. Chem., 2009, 19, 6023.

25 J. Chomoucka, J. Drbohlavova, D. Huska, V. Adam, R. Kizek and J. Hubalek, Pharmacol. Res., 2010, 62, 144-149.

26 Y. Xu, Y. Qin, S. Palchoudhury and Y. Bao, Langmuir, 2011, 27, 8990-8997.

27 A. K. Gupta and S. Wells, IEEE Trans. Nanobiosci., 2004, 3, 66-73.

28 I. Hussain, S. Graham, Z. X. Wang, B. Tan, D. C. Sherrington, S. P. Rannard, A. I. Cooper and M. Brust, J. Am. Chem. Soc., 2005, 127, 16398-16399.

29 Z. Wang, B. Tan, I. Hussain, N. Schaeffer, M. F. Wyatt, M. Brust and A. I. Cooper, Langmuir, 2006, 23, 885-895.

30 B. Xiao, Y. Wan, X. Y. Wang, Q. C. Zha, H. M. Liu, Z. Y. Qiu and S. M. Zhang, Colloids Surf., B, 2012, 91, 168-174.

31 Y. Yin and A. P. Alivisatos, Nature, 2005, 437, 664-670.

32 M. Mikhaylova, D. K. Kim, N. Bobrysheva, M. Osmolowsky, V. Semenov, T. Tsakalakos and M. Muhammed, Langmuir, 2004, 20, 2472-2477.

33 C. B. Murray, D. J. Norris and M. G. Bawendi, J. Am. Chem. Soc., 1993, 115, 8706-8715.

34 E. Amstad, S. Zurcher, A. Mashaghi, J. Y. Wong, M. Textor and E. Reimhult, Small, 2009, 5, 1334-1342.

35 J. Xie, C. Xu, N. Kohler, Y. Hou and S. Sun, Adv. Mater., 2007, 19, 3163-3166.

36 K. Nawara, J. Romiszewski, K. Kijewska, J. Szczytko, A. Twardowski, M. Mazur and P. Krysinski, J. Phys. Chem. C, 2012, 116, 5598-5609. 
37 L. Sun, C. Huang, T. Gong and S. Zhou, Mater. Sci. Eng., C, 2010, 30, 583-589.

38 Y.-J. Zhang, Y.-W. Lin, C.-C. Chang and T.-M. Wu, Synth. Met., 2010, 160, 1086-1091.

39 H. Qu, D. Caruntu, H. Liu and C. J. O'Connor, Langmuir, 2011, 27, 2271-2278.

40 D. L. A. de Faria, S. Venâncio Silva and M. T. de Oliveira, J. Raman Spectrosc., 1997, 28, 873-878.

41 K. N. Jallad and D. Ben-Amotz, Mater. Sci. Technol., 2001, 17, 1479-1486.

42 U. Schwertmann and R. M. Cornell, in Iron Oxides in the Laboratory, Wiley-VCH Verlag GmbH, 2007, pp. 27-54.

43 A. Demortière, P. Panissod, B. P. Pichon, G. Pourroy, D. Guillon, B. Donnio and S. Bégin-Colin, Nanoscale, 2011, 3, 225.
44 N. Singh, G. J. S. Jenkins, R. Asadi and S. H. Doak, Nano Reviews, 2010, 1, 5358.

45 Y. Qin, L. Sun, X. Li, Q. Cao, H. Wang, X. Tang and L. Ye, J. Mater. Chem., 2011, 21, 18003-18010.

46 S. Kayal and R. V. Ramanujan, Mater. Sci. Eng., C, 2010, 30, 484-490.

47 M. Dalmark and H. H. Storm, J. Gen. Physiol., 1981, 78, 349364.

48 H. C. Arora, M. P. Jensen, Y. Yuan, A. Wu, S. Vogt, T. Paunesku and G. E. Woloschak, Canc. Res., 2012, 72, 769-778.

49 T. Skovsgaard and N. I. Nissen, Pharmacol. Ther., 1982, 18, 293-311.

50 Y.-J. Gu, J. Cheng, C. W.-Y. Man, W.-T. Wong and S. H. Cheng, Nanomedicine: NBM, 2012, 8, 204-211. 